\title{
USO DE ÍNDICES HEMATIMÉRRICOS NO DIAGNÓSTICO DIFERENCIAL DE ANEMIAS MICROĆTIICAS: UMA ABORDAGEM A SER ADOTADA?
}

\author{
Murilo Rezende Melo*, Maria Cristina Purini, Rodolfo D. Cançado, Fernando Kooro, Carlos Sérgio Chiattone \\ Trabalho realizado na Disciplina de Hematologia e Hemoterapia da Faculdade de Ciências Médicas da Santa Casa \\ de São Paulo, SAE Laboratório Médico e Serviço de Patologia Clínica da Santa Casa de São Paulo, S. Paulo, SP.
}

RESUMO - O diagnóstico diferencial das anemias microcíticas é complexo e sua investigação laboratorial, de custo elevado. 0 uso de índices hematimétricos para racionalizar a abordagem diagnóstica tem sido proposto para contornar essa problemática.

OBjetivo. Avaliar a utilidade diagnóstica dos índices hematimétricos nas anemias microcíticas, de modo prospectivo, em hospital geral de alta complexidade.

Métodos. Foram analisados 2278 hemogramas realizados nos nossos serviços. Baseados em eletroforese de hemoglobina e ferritina, estratificamos 52 pacientes adultos com anemia microcítica em três grupos: Anemia ferropriva (AF; $n=26$ pacientes), BetaTalassemia Menor (BTM; $n=17)$ e Anemia não-ferropriva e nãobeta-Talassemia (ANFNT; $n=9$ ). Avaliamos 0 uso dos seguintes índices hematimétricos na discriminação dos três grupos, por análise de variância e curvas ROC: RBC, VCM, HCM, RDW, índices de England e de Green.
RESULTADOS. Nenhum dos índices permitiu a separação integral dos três grupos. Determinamos valores de corte para cada um dos índices e calculamos sensibilidade $(S)$, especificidade(E), valores preditivos positivo e negativo e eficácia, em função de sua melhor discriminação. A discriminação de BTM foi melhor realizada pelo RBC acima de 5 milhöes $/ \mu$, com $S=82,3 \%$ e $E=82,8 \%$; enquanto a anemia ferropriva, pelo RDW acima de $16 \%$, mas com $S$ de apenas $69,2 \%$ e $E=80,7 \%$.

CONCLUSÖES. A anemia ferropriva apresenta difícil diagnóstico presuntivo pelos índices, devendo ser realizada confirmação laboratorial. Valores elevados de RBC em anêmicos devem fazer suspeitar de traço talassêmico, sendo recomendável confirmação diagnóstica.

UNITERMOS: Hemograma. Índices hematimétricos. Talassemia menor. Anemia ferropriva. Sensibilidade. Especificidade.

\section{INTRODUÇÃO}

A deficiência de ferro é a causa mais freqüente de anemia em todo o mundo, constituindo-se em grave problema de saúde pública em nosso meio. Por outro lado, em muitas partes do mundo, a freqüência de betatalassemia como causa de anemia microcítica é menor apenas do que a frequêencia de anemia ferropriva. Apesar das formas graves de betatalassemia serem facilmente diagnosticadas, formas mais leves podem ser interpretadas e tratadas como anemia ferropriva, ${ }^{\prime}$.

O diagnóstico diferencial das anemias microcíticas é complexo e sua investigação laboratorial de custo elevado. O uso de índices hematimétricos para racionalizar a abordagem diagnóstica tem sido proposto para contornar essa problemática. Entretanto, seu emprego tem sido melhor documentado em estudos

*Correspondencia:

R. Maestro Elias Lobo, 93 - Jd. Paulista

São Paulo - SP - CEP01433-000 - Tel 3889-7200

Fax: 3884-6716-E-mail: murilomelo@uol.com.br retrospectivos, com grupos de anemia ferropriva e beta-talassemia menor ${ }^{3,4,5,6}$.

Várias diferenças entre a distribuição de tamanhos dos eritrócitos (RDW) têm sido descritas, com maior anisocitose em casos de anemia ferropriva ${ }^{7,8,9,10}$. Também tem sido observado que para um mesmo grau de anemia, os eritrócitos na beta-talassemia são mais microcíticos do que na anemia ferropriva. Conseqüentemente, o volume corpuscular médio(VCM) tende a ser menor e contagem de eritrócitos (RBC) maior em casos de betatalassemia. Índices complexos, que incorporam os parâmetros do hemograma em fórmulas matemáticas, têm sido utilizados ${ }^{3,4,6}$.

O objetivo deste trabalho foi avaliar a utilidade diagnóstica dos índices hematimétricos de modo prospectivo em hospital geral de alta complexidade.

\section{MÉTodos}

Foram analisados 2278 hemogramas de pacientes internados e ambulatoriais, realizados consecutivamente em nosso serviço, du- rante seis dias. As amostras foram colhidas a vácuo em tubos com EDTA-K3 (Vacutainer, Becton-Dickinson). Os exames foram todos realizados em um mesmo aparelho automatizado, seguindo-se o procedimento do fabricante (Abbott, Cell-Dyn 3000), com controles internos, (do fabricante e repetição de uma mesma amostra a cada 20 testes) e externo (PELM-Programa de Excelência para Laboratórios Médicos, Control-Lab).

A análise dos dados baseou-se na listagem dos resultados do equipamento. Todo teste com dosagem de hemoglobina $(\mathrm{Hb})$ inferior a $13 \mathrm{~g} / \mathrm{dL}$ e determinação do VCM inferior a 80 $\mathrm{fL}$ foi selecionado, sendo acessado o Sistema de Informática Laboratorial (SIL) para verificação dos dados: sexo, idade, número do prontuário e existência de amostra de soro no laboratório.

Utilizamos como critério diagnóstico de anemia os valores de $\mathrm{Hb}$ inferiores a $\mathrm{I} 3 \mathrm{~g} / \mathrm{dL}$ para homens e $12 \mathrm{~g} / \mathrm{dL}$ para mulheres, ambos acima de 14 anos de idade. Para as demais faixas etárias, utilizou-se valores de referência 
da literatura para diagnóstico de anemia" . Não consideramos as faixas etárias onde os valores de hemoglobina de $13 \mathrm{~g} / \mathrm{dL}$ ou superior possam ser critério de anemia. Microcitose foi definida como VCM inferior a $80 \mathrm{fL}$.

Encontramos 343 testes (15\%) que apresentaram anemia microcítica, considerando os valores de referência para a idade. Destes, 134 ( $5,9 \%$ do total) eram de pacientes acima de 14 anos. Amostras concomitantes de soro, solicitadas para a realização de testes diversos, foram encontradas em 52 pacientes, que foram objeto de nosso estudo.

A dosagem de ferritina foi realizada por enzima-imunoensaio com micropartículas (MEIA, AxSym, Abbott), utilizando-se amostra de soro. A amostra do hemograma foi utilizada para realização de eletroforese de hemoglobina em $\mathrm{pH}$ alcalino e dosagem de hemoglobina fetal pelas técnicas habituais ${ }^{12,13}$.

Estratificamos os casos, baseados nos dados laboratoriais, em três grupos:

Anemia ferropriva com eletroforese de hemoglobina normal eferritina inferior aos valores de referência para o sexo e idade(Homens: 20 - 250 $\mu \mathrm{g} / \mathrm{L} ;$ Mulheres: 10 - $120 \mu \mathrm{g} / \mathrm{L})^{14}$.

Beta-talassemia menor quando a eletroforese de hemoglobina apresentava elevação da proporção de hemoglobina $\mathrm{A} 2$ (>3,5\%), mas com ferritina normal.

Anemia não-ferropriva e não-beta-talassemia quando a eletroforese de hemoglobina ea dosagem de ferritina encontravamse normais.

Não encontramos casos mistos, isto é, pacientes com eletroforese de hemoglobina compatível com beta-talassemia menor e dosagem de ferritina inferior ao valor de referência.

Avaliamos o uso dos seguintes índices hematimétricos na discriminação dos três grupos, por análise de variância (programa computacional SigmaStat, Sigma) e curvas ROC (Relative Operating Characteristic; programa SPSS v. I0): RBC, VCM, HCM (Hemoglobina Corpuscular Média), RDW, índice de England (VCM - RBC - $(5 * \mathrm{Hb})$ $3,4)$ e índice de Green $\left(\mathrm{VCM}^{\wedge} 2\right.$ * (RDW/ 100) * Hb). Adotou-se alfa $=0,05$ e menos como nível de significância estatística. Uma vez determinada a significância da análise estatística, foi determinado o nível de corte para cada índice e calculados sensibilidade, especificidade, valores preditivos positivo e negativo e eficácia.

\begin{tabular}{lccccc}
\hline \multicolumn{5}{c}{ Tabela I - Desempenho de índices hematimétricos em anemias microciticas } \\
\hline & $\begin{array}{c}\text { Sensibilidade } \\
(\%)\end{array}$ & $\begin{array}{c}\text { Especificidade } \\
(\%)\end{array}$ & $\begin{array}{c}\text { Valor Preditivo } \\
\text { Positivo(\%) }\end{array}$ & $\begin{array}{c}\text { Valor Preditivo } \\
\text { Negativo(\%) }\end{array}$ & $\begin{array}{c}\text { Eficácia } \\
(\%)\end{array}$ \\
$\begin{array}{l}\text { RBC }>5 \text { milhôes } / \mathrm{mL} \\
\text { (Talassemiavs. Outros) }\end{array}$ & 82,3 & 82,8 & 70 & 91 & 82,6 \\
$\begin{array}{l}\text { England }<6 \\
\text { (Talassemiavs. Outros) }\end{array}$ & 82,3 & 82,8 & 70 & 91 & 82,6 \\
$\begin{array}{l}\text { Green }<72 \\
\text { (Talassemiavs. Outros) }\end{array}$ & 76,4 & 85,7 & 72 & 88 & 82,6 \\
$\begin{array}{l}\text { VCM }<68,5 f \mathrm{fL} \\
\text { (Talassemiavs. Outros) }\end{array}$ & 76,5 & 74,3 & 59,1 & 86,7 & 75 \\
RDW $>16 \%$ & 69,2 & 80,7 & 78 & 72 & 75 \\
(Ferroprivavs. Outros) & & & & & \\
\hline
\end{tabular}

\section{Resultados}

Nenhum dos índices permitiu a separação integral dos três grupos, a saber: Anemia ferropriva (AF; $n=26$ pacientes), Betatalassemia menor (BTM; $n=17$ ) e Anemia não-ferropriva enão-beta-talassemia (ANFNT; $\mathrm{n}=9$ ). Buscamos, assim, a capacidade de cada índice em melhor separar um dos grupos dos demais. Determinamos valores de corte para cada um dos índices e calculamos sensibilidade, especificidade, valores preditivos positivo e negativo e eficácia, em função de sua melhor discriminação (Tabela I).

A discriminação de BTM foi melhor realizada pelo $\mathrm{RBC}$ acima de 5 milhões $/ \mu \mathrm{L}$ (igualada pelo índice de England $<6$ ), com sensibilidade de $82,3 \%$ e especificidade de $82,8 \%$; enquanto a anemia ferropriva, pelo RDW acima de $16 \%$, mas com sensibilidade de apenas $69,2 \%$ e especificidade de $80,7 \%$.

O grupo ANFNT não foi estatisticamente diferenciado dos demais grupos por nenhum índice, apresentando grande sobreposição de dados. A análise dos prontuários destes pacientes revelou que quatro dos nove pacientes haviam sido consultados apenas uma vez e ainda não apresentavam diagnóstico etiológico da anemia. Os cinco pacientes restantes apresentavam anemia falciforme, esclerodermia, adenocarcinoma de pulmão, adenocarcinoma de próstata e uma era gestante com necessidade transfusional. A mediana das dosagens de ferritina deste grupo foi de 137,5 $\mu \mathrm{g} / \mathrm{L}$ ( I 7 a $994 \mu \mathrm{g} /$ $\mathrm{L})$, clinicamente semelhante à mediana das dosagens no grupo com BTM que foi $89 \mu \mathrm{g} /$ $\mathrm{L}(12$ a $1094 \mu \mathrm{g} / \mathrm{L})$.

\section{Discussão}

As anemias microcíticas são real problema de saúde em nosso meio, ocorrendo em I5\% dos pacientes atendidos em nosso serviço. $A$ anemia ferropriva confirmou-se como a mais importante causa de anemia microcítica, mas observou-se um número de casos de BTM superior ao esperado. Após minuciosa verificação dos dados no SIL, pudemos observar que eram, em sua maioria, casos acompanhados pelo Serviço de Hematologia, podendo ter proporcionado certa tendenciosidade para mais na prevalência de BTM na amostra.

Utilizamos a dosagem de ferritina, com seus valores infranormais, para o diagnóstico de anemia ferropriva. Entretanto, esta proteína sofre elevação nas reações de fase aguda da inflamação, podendo causar resultados falsonegativos (pacientes aqui definidos como ANFNT poderiam ter AF).

Encontramos a elevação do RDW (acima de 16\%) como melhor índice discriminador de anemia ferropriva, o que é explicado pela coexistência de eritrócitos no sangue periférico produzidos em estágios progressivos da deficiência de ferro, causando mistura de células normocíticas e progressivamente microcíticas. $\mathrm{Na} B T M$, não ocorre flutuação da desordem na produção de hemoglobina e a medula óssea produz uma população mais uniforme de eritrócitos ${ }^{10,15,16}$. A sensibilidade do RDW no diagnóstico de $A F(69,2 \%)$ deve ser considerada insuficiente para utilização deste índice como teste de triagem, mas sua especificidade pode ser considerada razoável, de modo a permitir, em conjunto com dados clínicos, boa confiabilidade diagnóstica para AF. 
Melo MR et AL.

O diagnóstico de BTMé, ocasionalmente, negligenciado pelo clínico ao deparar-se com microcitose e níveis discretos de anemia. A contagem de eritrócitos acima de 5 milhões/ $\mu \mathrm{L}$ é índice bastante útil para triagem destes casos, com sensibilidade e especificidade adequadas. $O$ índice de England apresentou desempenho equivalente, mas a complexidade de seu cálculo não justifica sua implantação.

A condição de ANFNT apresentou grande sobreposição de dados com os outros dois grupos, não podendo ser diferenciada por nenhum índice. Esta observação é importante ao se considerar os dados de trabalhos anteriores, que compararam apenas grupos conhecidos de BTM e AF e obtiveram, assim, níveis de sensibilidade e especificidade muito superiores aos aqui apresentados ${ }^{3,4,5,6}$.

\section{ConClusões}

Aanemia ferropriva apresenta dificuldades em seu diagnóstico presuntivo pelos índices hematimétricos, devendo ser realizada investigação laboratorial complementar para diagnóstico mais apropriado. Valores elevados de $\mathrm{RBC}$ em anêmicos devem fazer suspeitar de traço talassêmico, sendo recomendável confirmação diagnóstica.

\section{SUMmarY}

Are Red Blood Cells (RBC) indices VALUABLE ON DIFFERENTIATING MICROCYTIC ANEMIAS?

Differential diagnosis of microcyticanemia is a complex task, with considerable cost.

OBJECTIVE. To evaluate prospectively RBC indices as a diagnostic tool at a high complexity generalhospital.

MetHods. We analyzed 2278 blood cell counts from the core laboratory of our service and we found 343 (15\%) microcytic anemias. Concomitantserum samples werefound from 52 patients above 14 years-old, and ferritin measurement and hemoglobin electrophoresis were performed. We classified our patients in three groups: Iron-deficiency anemia (AF; 26 patients), beta-thalassemia minor (BTM; $n=17)$ and non-iron-deficiency-non-beta-thalassemia (ANFNT; $n=9$ ). Weevaluated the followingRBC indices for group discrimination, with variance and ROC curve analysis: $R B C, M C V, M C H$, $R D W$, England's index and Green's index.

RESULTS. None of the indices allowed complete discrimination of all groups. We have established limits for each of the indices, and sensitivity (S), specificity $(E)$, positive and negative predictive values and test efficiency were determined. BTM was better distinguished from the other groups by $R B C$ (above 5 millions $/ \mu \mathrm{L}$ ) and England's index $(<6)$, both with $S=82.3 \%$ and Eof $82.8 \%$.ARDWabove $16 \%$ was the best index of $A F$, with $S=69.2 \%$ and $E=80.7 \%$.

CONCLUSIONs. Presumptive diagnosis of $A F$ is troublesome when using RBCindices; diagnosis should be based on complete laboratory evaluation. Elevated RBClevels, specially above 5 millions/ $\mu \mathrm{L}$, inanemic patients with microcytosis are important indicatives of BTM, and confirmatory tests are suggested. [Rev Assoc Med Bras 2002; 48(3): 222-4]

KEY WorDs: Blood cell counts. RBC index. Thalassemia minor. Iron-deficiency anemia. Sensitivity. Specificity.

\section{REFERÊNCIAS}

I. Cunningham LO, Rising JA. Erytrocytic microcytosis: clinical implications in 100 patients. Am J Med Sci 1977; 273: I 49-55.

2. Pearson HA, O'Brien RT, Maclntosh S. Screening for thalassemia trait by electronic measurement of mean corpuscular volume. $\mathrm{N}$ Engl J Med I 973; 288:35I-3.

3. England JM, Frase PM. Differentiation of iron deficiency from thalassaemia trait by routine blood-count. Lancet 1973; I:449-452.

4. Green R, King R. A new red cell discriminant incorporating volume dispersion for differentiating iron deficiency anemia from thalassemia minor. Blood Cells 1 989; 15:48 I-95.

5. Lima CSP, Reis ARC, Grotto HZW, Saad STO,
Costa FF. Comparison of red cell distribution width and a red cell discriminant function incorporating volume dispersion for distinguishing iron deficiency from beta thalassemia trait in patients with microcytosis. São Paulo Med J 1996; I 14:1265-9.

6. Eldibany MM, Totonchi KF, Joseph NJ, Rhone $D$. Usefulness of certain red blood cell indices in diagnosing and differentiating thalassemia trait from iron-deficiency anemia. Am J Clin Pathol I 999; I I I:676-82.

7. Bessman JD, Gilmer PR, Gardner FH. Improved classification of anemias by MCV and RDW. Am J Clin Pathol I 983; 80:322-6.

8. Karnad A, Poskitt TR. The automated complete blood cell count. Arch Intern Med 1985; 145: 1270-2.

9. McClure S, Custer E, Bessman JD. High RDW is the earliest predictor of iron deficiency. Blood I 983; 62(Suppl I):5 Ia.

10. Miguel A, Linares M. Miguel A, Miguel-Borja JM. Red cell distribution width analysis in differentiation between iron deficiency and thalassemia minor. Acta Haematol 1988; 80:59.

II. Geaghan SM. Hematologic values and appearances in the healthy fetus, neonate, and child. Clin Lab Med 1999; 19: I - 37.

12. Schneider RG. Identification of hemoglobins by elctrophoresis. In: Schmidt RM, et al., editors. The detection of hemoglobinopathies. Cleveland: CRC Press; 1974.

13. Kleihauer E. Determination of fetal hemoglobin: elution technique. In: Schmidt RM, editor. The detection of hemoglobinopathies. Cleveland: CRC Press; 1974.

14. Fairbanks VF, Klee, GG. Aspectos bioquímicos da hematologia. In: Burtis CA, Ashwood ER, editors. Tietz fundamentos de química clínica. 4a. ed. Rio de Janeiro: Guanabara Koogan; 1998. p.68-706.

15. Johnson CS, Tegos C, Beutler E. Thalassemia minor: routine erytrocyte measurements and differentiation from iron deficiency. Am J Clin Pathol I 983; 80:31-6.

16. Ghionni H, Miotti TC, Camandona V. Routine erytrocyte measurements and differentiation of thalassemia minor from iron deficiency. Minerva Med I 985; 76: I I43-8.

Artigo recebido: 30/10/2000

Aceito para publicação: 20/06/200I 